\title{
Diseño de un Sistema Tutor Inteligente Multiagente 1
}

\author{
Elizabeth Jiménez Rey ${ }^{1}$, María Delia Grossi ${ }^{1}$, Arturo Carlos Servetto ${ }^{1}$ \\ Félix Anibal Vallejos ${ }^{1,2}$, Gregorio Perichinsky ${ }^{1}$ \\ ${ }^{1}$ \{ejimenez,mdgrossi,aserve,gperi\}@mara.fi.uba.ar \\ ${ }^{2}$ felix_vallejos@yahoo.com.ar \\ ${ }^{1,2}$ Departamento de Computación - $4^{\mathrm{o}}$ Piso - Ala Sur. - Laboratorio de Sistemas Operativos y Bases de \\ Datos - Facultad de Ingeniería - Universidad de Buenos Aires - Paseo Colón No 850 - (1063) Buenos \\ Aires - Argentina - Teléfono: (54 11) 4343-1177 (int. 140/145) - FAX: (54 1) 4331-0129
}

\begin{abstract}
Resumen
Se presentó en una publicación previa la primera etapa de una línea de investigación dentro del Proyecto de Investigación Acreditado I015 "Manufactura Integrada por Computadora en Sistemas Complejos para el Desarrollo Social, Industrial y de Tecnología". Se ilustraron en un marco teórico los temas básicos que pretende aunar dicho trabajo: los Sistemas Tutores Inteligentes (STI) y los Sistemas Multiagentes (SMA). La creación de Sistemas Inteligentes Educativos (SIE) se enfoca más como una herramienta complementaria de la enseñanza y del aprendizaje que permite aumentar la calidad del aprendizaje, que como una herramienta que sustituye en sí todo un sistema clásico de enseñanza y de aprendizaje. En este trabajo se describe los aspectos esenciales que se deben considerar en el modelado de un sistema tutor inteligente utilizando la tecnología de agentes y se propone el diseño de uno en particular, especialmente su modelo de datos.
\end{abstract}

Palabras-clave: Informática Educativa, Tecnología Informática, Aplicación Educativa y Cognitiva. Multiagentes.

\begin{abstract}
In a previous paper, we have presented the first step o fan investigation line within the Accredited Research Project IO15 "Computer Integrated Manufacturing In Complex Systems for the Social Industrial and Technology Development". We have showed, in a theoretical view the basic subjects of the paper: The Tutor Intelligent Systems (STI) and The Multiagents Systems (SMA). The development of the Educational Intelligent Systems (SIE) is more focused as a teaching and learning complementary tool which allow to increase the learning quality, than as a tool which replaces the classic teaching and learning system. In this paper we describe the essential items we must consider for modeling a tutor intelligent system using the agent technology and we propose the design of a one particulary system, specially its date model.

Key-words: Informatic Education, Informatic Technology, Education and Cognitive Application, Multiagents.
\end{abstract}

\section{Introducción}

Un Sistema Multiagente (SM) es un conjunto de agentes (sean estos procesos, personas, moléculas) autónomos que no comparten memoria, ni realizan elecciones en forma sincrónica, pero interactúan entre si intercambiando información, masa o energía, así como compartiendo recursos (agua, petróleo, CPU). La modelización de un SM debe contemplar, al menos: los mecanismos de comunicación global entre los agentes; los mecanismos de protección global, las interfaces de comunicación y los mecanismos para la optimización del uso de los recursos.

El carácter del presente proyecto exige el uso de modelos matemáticos aplicables tanto al caso de sistemas informáticos (redes, INTERNET) como al de sistemas sociales. Para satisfacer este punto se pueden utilizar modelos derivados de la mecánica estadística, que se describen brevemente a modo de ejemplo.

Tal como expresamos anteriormente, el modelo del SM debe contener la descripción de mecanismos básicos que contemplen la interacción de muchos agentes intentando resolver una variedad de problemas en forma cooperativa. Cada agente tiene una visión local única del problema, y al mismo tiempo debe interactuar satisfactoriamente con otros agentes con el fin de encontrar soluciones globales a los problemas propuestos utilizando estrategias de problema/solución buscando con que otros agentes ellos pueden colaborar. Como la adecuación de estas opciones puede cambiar, si 
los agentes hacen elecciones en presencia de conocimientos tardíos e imperfectos sobre el estado del sistema, su dinámica puede hacerse extremadamente compleja, dando lugar a regimenes caóticos. Se considera un modelo basado en que los agentes hacen opciones locales con información imperfecta para lograr sus tareas. Este modelo utilizado para inteligencia artificial distribuida se construye por analogía con organizaciones biológicas y humanas similares. Las opciones de los agentes pueden estar entre estrategias $\mathrm{u}$ otros agentes con los cuales interactuar. Los agentes seleccionan independientemente y asincrónicamente entre las opciones disponibles el que tiene el payoff percibido más alto. Estos payoffs reflejan el beneficio real obtenido entre varias opciones, como el tiempo exigido para completar una tarea, exactitud de la solución, etc. Porque los resultados de varias elecciones pueden depender de lo que los otros agentes hacen, estos payoffs dependerán típicamente de las elecciones hechas por otros agentes. Para mayor simplicidad al analizar la conducta global de sistemas grandes nosotros tomamos, el payoff $\mathbf{g}_{\mathbf{r}}$. para usar el recurso $\mathbf{r}$ para depender del número de agentes que ya lo usan, más bien exactamente qué agentes son éstos. Aunque simple, esta dependencia captura algunas propiedades generales. Por ejemplo, en un ambiente completamente competitivo, el payoff para usar un recurso particular tiende a disminuir cuantos más agentes hagan uso de él. En cambio en situaciones cooperativas, el payoff de un recurso debería aumentar cuantos más agentes hacen uso de él. La información imperfecta sobre el estado del sistema hace que el payoff percibido de cada agente difiera del valor real, diferencia que aumenta cuando hay más incertidumbre en la información disponible a los agentes. Este tipo de incertidumbre concisamente captura el efecto de muchas fuentes de errores como algunos bichos de programas, heurísticas evaluando opciones incorrectamente, errores en la comunicación y errores interpretando datos sensitivos. Específicamente, los payoffs percibidos se toman normalmente para ser distribuidos, con desviación estándar $\sigma$, alrededor de sus valores correctos. Además, retrasos de información hacen que el conocimiento de cada agente del estado del sistema esté fuera de fecha (desactualizado).

Aunque para simplicidad consideraremos el caso en el que todos los agentes tienen el mismo retraso eficaz, incertidumbre, y preferencias para el uso del recurso, debemos mencionar que el mismo rango de conductas también se encuentra en una situación más general. Como un ejemplo específico, consideramos el caso de dos recursos, así el sistema puede ser descrito por la fracción $\mathbf{f}$ de agentes que están usando el recurso 1 en cualquier momento dado. Su dinámica es gobernada entonces por:

$$
\frac{d f}{d t}=\alpha(\rho-f)
$$

donde $\boldsymbol{\alpha}$ es la proporción a la que los agentes reevalúan su opción del recurso y $\rho$ es la probabilidad de que un agente prefiera el recurso 1 sobre el 2 cuando hace una selección. Generalmente, $\boldsymbol{\rho}$ es una función de $\boldsymbol{f}$ a través de los payoffs de densidad dependiente. En términos de payoffs e incertidumbre, tenemos

$$
\rho=\frac{1}{2}\left(1+\exp \left(\frac{G_{1}(f)-G_{2}(f)}{2 \sigma}\right)\right)
$$

donde $\boldsymbol{\sigma}$ cuantifica la incertidumbre. Esta definición captura el simple requisito de que un agente es más propenso a preferir un recurso cuando su payoff es relativamente grande. Finalmente, los retrasos en información son modelados suponiendo que los payoffs que entran en $\boldsymbol{\rho}$ en el tiempo $\boldsymbol{t}$ son los valores que ellos tenían en un momento retardado $\mathbf{t}-\boldsymbol{\tau}$. Cuando los retrasos e incertidumbre son bastante pequeños, el sistema converge en un punto de equilibrio cerca del óptimo asequible por un controlador central omnisciente. Como la información disponible a los agentes se vuelve más adulterada, el punto de equilibrio se aleja más del valor óptimo. Con retrasos crecientes, el equilibrio se vuelve inestable, llevando a la conducta oscilatoria y caótica. En estos casos, el número de agentes que usan recursos particulares continúa variando por lo que el sistema pasa un tiempo relativamente pequeño cerca del valor óptimo, con la caída consecuente, en su performance global. Las formas de control de las situaciones caóticas, abre numerosas variantes de investigación. Un modelo utilizado para Inteligencia Artificial Distribuída se puede describir especificando la fracción de agentes, $\boldsymbol{f}_{\text {rs }}$ de un tipo dado $\boldsymbol{s}$ usando un recurso dado $\boldsymbol{r}$ en un momento particular. También definiremos la fracción total de agentes que usan un recurso de un tipo particular como:

$$
\begin{aligned}
& f_{r}^{r c s}=\sum_{s} f_{r s} \\
& f_{s}^{t i p o}=\sum_{r} f_{r s}
\end{aligned}
$$

respectivamente. Como se mencionó previamente, el efecto red de compensar la actuación es aumentar la fracción de agentes de actuación altamente favorable. Si $\gamma$ es la proporción a la que la actuación es compensada, entonces la Eq. (1) se refuerza con un término extra que corresponde a este mecanismo de la compensación.

Esto da:

$$
\frac{d f_{r s}}{d t}=\alpha\left(f_{s}^{t i p o} \rho_{r s}-f_{r s}\right)+\gamma\left(f_{r}^{r c s} \eta_{s}-f_{r s}\right)
$$

donde el primer término es análogo a lo dicho en la teoría anterior, y el segundo término incorpora el efecto 
de las compensaciones en la población. En esta ecuación $\rho_{r s}$ es la probabilidad de que un agente de tipo $\boldsymbol{s}$ preferirá el recurso $\boldsymbol{r}$ cuando realiza una opción, y $\boldsymbol{\eta}_{\mathbf{s}}$ es la probabilidad de que nuevos agentes serán de tipo $s$, que tomamos como proporcionales al payoff actual asociado con agentes de tipo s. Como antes $\boldsymbol{\alpha}$ denota la proporción a la que los agentes hacen elecciones de recursos y la interpretación detallada de $\gamma$ depende del particular mecanismo de compensación involucrado. Como la fracción total de agentes de todos los tipos debe ser uno, una forma simple de la condición de normalización puede obtenerse si uno considera el payoff relativo que es dado por ${ }^{1}$ :

El numerador es el payoff real recibido por agentes de tipo s dado su corriente uso del recurso y el denominador es el payoff total para todos los agentes en el sistema, ambos, normalizados al número total de agentes en el sistema. Sumando Eq. 4 sobre todos los recursos y los tipos dan:

$$
\begin{aligned}
\frac{d f_{r}^{r c s}}{d t} & =\alpha\left(\sum_{s} f_{s}^{i y p c} \rho_{r s}-f_{r}^{r c s}\right) \\
\frac{d f_{s}^{i y p c}}{d t} & =\gamma\left(\eta_{s}-f_{s}^{i y p c}\right)
\end{aligned}
$$

lo cual describe la dinámica de uso del recurso global y la distribución de tipos de agentes, respectivamente. Esto implica que aquellos tipos de agentes que reciben más del payoff promedio(es decir tipos para los cuales $\boldsymbol{\eta}_{\mathbf{s}}>\boldsymbol{f}_{\boldsymbol{s}}^{\text {iypc }}$ aumentarán en el sistema a expensas de los tipos de baja performance. Los payoffs reales sólo pueden compensar tipos de agentes existentes. Así para introducir nuevas variaciones en la población se necesita un mecanismo adicional (ej. correspondiente a la mutación en algoritmos genéticos o de aprendizaje). [5]

\section{Metodología}

\subsection{Antecedentes}

La idea de aprovechar herramientas informáticas en la enseñanza se remonta a los años 50. Los sistemas de

\footnotetext{
1 esta forma asume payoffs positivos, ej. ellos podrían ser
} proporciones de crecimiento. Si los payoffs pueden ser negativos (ej. ellos son cambios de dinero en un sistema económico), uno puede usar en cambio la diferencia entre los payoffs reales y su valor mínimo $\mathrm{m}$. Como $\eta_{\mathrm{s}}$ debe sumar 1 , esto dará :

$$
\eta_{s}=\frac{\sum_{r} f_{r s} G_{r}}{\sum_{r} f_{r}^{r c s} G_{r}}
$$

qué se reduce al caso anterior cuando: $\mathrm{r} . \mathrm{n}=0$. enseñanza tradicionales desarrollados antes de la aparición de los primeros STI se conocen con el nombre de EAC (Enseñanza Asistida por Computadora, O CAI: Computer-Assisted Instruction). Pero no será hasta los años 80 cuando la enseñanza asistida por computadora recobre un especial interés gracias a las técnicas de la Inteligencia Artificial (IA). En esa época surgen los denominados STI con la vocación clara de desarrollar procesos de enseñanza adaptados a los diferentes usuarios/estudiantes. [8]

Un STI es un sistema de enseñanza asistida por computadora, que utiliza técnicas de IA, principalmente para representar el conocimiento y dirigir una estrategia de enseñanza. Es capaz de comportarse como un experto, tanto en el dominio de conocimiento que enseña (mostrando al alumno cómo aplicar dicho conocimiento), como en el dominio pedagógico (donde es capaz de diagnosticar la situación en la que se encuentra el estudiante y de acuerdo a ello ofrecer una acción o solución que le permita progresar en el aprendizaje). [6]

La creación de Sistemas Inteligentes Educativos (SIE) se enfoca más como una herramienta complementaria de la enseñanza y del aprendizaje que permite aumentar la calidad del aprendizaje, que como una herramienta que sustituye en sí todo un sistema clásico de enseñanza y de aprendizaje.

Los Sistemas EAC han ido evolucionando de una manera notoria. En los años 50 aparecieron los primeros sistemas de enseñanza, llamados programas lineales. Estos programas se caracterizaban por mostrar el conocimiento de una manera lineal. Es decir, ningún factor podía cambiar el orden de enseñanza establecido en su momento por el programador. En el desarrollo de una sesión de enseñanza no se tiene en cuenta la aptitud del alumno. Los sucesores de los programas lineales en el campo de la enseñanza asistida por ordenador, fueron los programas ramificados (Crowed 1959). Éstos tenían un número fijo de temas, al igual que los programas lineales; sin embargo, se diferenciaban por la capacidad de actuar según la respuesta del alumno. A finales de los años sesenta y principios de los años setenta (19671971) surgieron los sistemas generativos (también llamados sistemas adaptativos). Éstos van asociados a una nueva filosofía educativa que manifiesta que "los alumnos aprenden mejor enfrentándose a problemas de dificultad adecuada, que atendiendo a explicaciones sistemáticas", es decir, adaptando la enseñanza a sus necesidades. Estos sistemas son capaces de generar un problema acorde al nivel de conocimiento del alumno, construir su solución y diagnosticar la respuesta del alumno. Como evolución de los sistemas EAC y con la incorporación de técnicas de IA aparecieron los STI.[8]

Se les atribuye "inteligencia" a estos sistemas por su capacidad de resolver los problemas que presenta a los estudiantes y de explicar cómo lo hacen. Al igual que los sistemas de EAC tradicionales, permiten un mayor grado de individualización en la instrucción; en particular, un STI, relaciona la instrucción con el entendimiento de las metas y creencias del estudiante. En un sistema de EAC no inteligente, el orden y plan de interacción están más bien predefinidos; mientras que 
en un STI, se usan técnicas de IA tales como planificación, optimización y búsquedas, dejando que el sistema decida "inteligentemente" el orden de presentación del contenido al alumno. [6]

Los STI se caracterizan por representar separadamente la materia que se enseña (modelo del dominio) y las estrategias para enseñarla (modelo pedagógico). Por otro lado, caracterizan al alumno (a través de un modelo del estudiante) para procurar una enseñanza individualizada. Además, de una manera cada vez más necesaria y al igual que cualquier software que se comunica con usuarios, la interfaz de comunicación se corresponde con un módulo bien planificado, de fácil manipulación y que favorece el proceso de comunicación tutor-alumno. [8]

\subsection{Arquitectura}

Los STI poseen una estructura modular donde cada módulo además de desempeñar una función específica dentro de la arquitectura, está interrelacionado y en sincronía con los demás. [3]

\section{Módulo del Alumno}

Almacena informaciones específicas para cada estudiante de forma individual. Como mínimo, este módulo debe mantener un histórico sobre cómo está trabajando el estudiante con el material en cuestión. Es interesante también mantener registro sobre los errores del estudiante. El módulo del alumno representa el conocimiento y las habilidades cognitivas del alumno en un momento dado. Contiene una representación del estado del conocimiento del alumno en el momento que interactúa con el STI. A partir de ese modelo y del contenido representado en la base del dominio, el sistema debe ser capaz de inferir la mejor estrategia de acción a ser utilizada para cada alumno. [5]

\section{Módulo Tutorial}

El módulo pedagógico ofrece una metodología para el proceso de aprendizaje. Posee el conocimiento sobre las estrategias y tácticas para seleccionarlas en función de las características del alumno y determina la manera en que la información será representada. Ejecuta el diagnóstico del conocimiento del alumno [7]. Las entradas de este módulo son ofrecidas por el Módulo del Alumno.

\section{Módulo del Dominio}

El módulo del dominio almacena la información que el tutor está enseñando. El modelado del conocimiento a ser transferido es de gran importancia para el éxito del sistema como un todo. Se debe procurar una representación del conocimiento que esté preparada para el crecimiento incremental del dominio. Este modelo sirve como base para la construcción del Modelo del Alumno [7].

\section{Interfaz}

Intermedia la interacción entre el tutor y el alumno. Presenta el material apropiado al nivel de entendimiento del alumno y mantiene la coherencia en las explicaciones [7].
Esta propuesta trajo grandes avances al modelado de ambientes educacionales pues separó el dominio de su forma de manipulación, permitiendo así que estrategias de enseñanza fuesen asociadas en función de las informaciones oriundas del modelado del alumno y relacionadas con que el dominio (contenido) es organizado.

La arquitectura básica de un STI incluye: consideraciones didácticas (cómo se enseña), características de los diferentes tipos de dominios (qué se enseña), propuestas diferenciadas según y a quién vayan dirigidos los sistemas (a quién se enseña). Los modelos del dominio, pedagógico y del estudiante son los específicos de un sistema de enseñanza inteligente. [5]

Esta arquitectura clásica fue ampliada a una arquitectura tripartida asociada al modelo de interacción que ocurre a lo largo de una sesión de trabajo entre el alumno y el ambiente. El módulo de dominio no es más una forma de representar las informaciones interrelacionadas sino un modelo de los aspectos de conocimiento sobre el dominio al que el alumno puede acceder durante las interacciones con el STI (Módulo de Situación). El módulo del estudiante ya no relaciona solamente las informaciones sobre el análisis de las interacciones del alumno con el dominio sino busca una contextualización mayor de estas interacciones en función de las acciones del alumno, el contexto en que ellas ocurren y la estructura cognitiva del alumno en ese momento (Módulo de Interacción). Y el módulo tutor dejó de ser el responsable de la selección del contenido y estrategias para convertirse en aquél que conduce al alumno de acuerdo con objetivos y desafíos educacionales que el ambiente proporciona al alumno (Módulo de Ofrecimientos).

La arquitectura de un STI puede servir como marco en un proceso de aprendizaje constructivo. Para clarificar esta afirmación es necesario reconsiderar los tres componentes tradicionales de los STI [5]:

- Los diseñadores de modelos con aprendizaje constructivo, diseñan el conocimiento del dominio según la naturaleza de las situaciones, contextos e interacciones posibles. Estas representaciones que pueden parecer similares a las del modelo del dominio de los STI, sin embargo tienen un propósito muy diferente. No hay descripciones de los objetos del dominio, sino descripciones de fuentes disponibles en determinadas situaciones. Desde esta perspectiva el modelo del dominio será un subconjunto de la noción más amplia de Modelo de Situación.

- $\quad$ Habitualmente el modelo del alumno de un STI se utiliza para analizar las interacciones del estudiante con referencia al modelo del dominio. El objetivo es detectar la falta de conocimiento o errores para establecer una posible intervención instruccional. Almacena informaciones específicas para cada estudiante de forma individual. Desde un punto de vista constructivista, el modelo particular del alumno, en cada momento, debe centrarse en los procesos interactivos del estudiante teniendo en cuenta sus acciones, el contexto en el que ocurre y sus estructuras cognitivas. Así, la idea de un Modelo de Procesos de Interacción es 
un superconjunto de la idea del modelo del estudiante en los STI.

- $\quad$ El módulo pedagógico determina los planes instruccionales, interpretando el modelo del estudiante con respecto al curriculum que refleja el modelo del dominio. Desde el punto de vista constructivista, el papel pedagógico del sistema no debe determinar los eventos instruccionales, sino proporcionar espacios de interacción al estudiante, basados en un modelo de ofrecimientos de situaciones potenciales. Así pues, se tiene una visión objetiva del dominio, desarrollada con un Modelo de Ofrecimientos, en términos de "ítems de conocimiento" que pueden aprenderse a través de eventos o situaciones particulares.

\subsection{Sistemas Multiagente}

Actualmente muchos de los ambientes de enseñanza y de aprendizaje computarizados en la modalidad de STI utilizan la tecnología de agentes en su proyecto. Este abordaje orientado a agentes, sustituye a los módulos de la arquitectura tradicional por una sociedad de agentes que trabajan en forma cooperativa usando diversas técnicas de IA e integrados como un SMA. [2]

La constante búsqueda de sistemas tomadores de decisiones determinó la aparición y el desarrollo de muchas áreas dentro de la IA. Una de estas áreas, desarrollada en los últimos años, se denomina Inteligencia Artificial Distribuida (IAD). Los SMA constituyen un área de investigación de la IAD. El planteo básico de la IAD partía del reconocimiento de que, hasta entonces, la IA se había centrado en emular las funciones cognitivas y de razonamiento del ser humano, de manera aislada de su contexto social, soslayando un componente crucial de la inteligencia humana: su dimensión social. La IAD buscaba explorar la dimensión social de la IA.

En los últimos años la IAD se tornó un dominio de investigación muy prometedor. Actualmente, miles de sistemas especialistas se basan en los conceptos de distribución de atribuciones para la especialización de tareas Según G. Weiss, la IAD, contraponiéndose a la inteligencia artificial clásica, basa su modelo de inteligencia en el comportamiento social transponiéndose el énfasis hacia las acciones e interacciones entre entidades denominadas agentes. [6]

\section{Fundamentos}

Los SMA constituyen un abordaje de la IAD cuyo foco de investigación son los agentes. Un agente puede ser definido en términos de sus propiedades fundamentales y debe poseer un cierto grado de autonomía para razonar y tomar decisiones por voluntad propia además de interactuar con otros agentes. Un agente debe poseer un cierto grado de independencia para resolver ya sea parcial o totalmente un problema. [4]

No existe una definición universalmente aceptada de agente. Si bien hay un consenso general sobre el hecho de que la autonomía es esencial en un agente, existe poca coincidencia en los demás aspectos del término. Parte de la dificultad se debe a que varios atributos asociados con agentes difieren en importancia para diferentes dominios. En consecuencia, para algunas aplicaciones, la habilidad de los agentes para aprender de su experiencia es un parámetro importante; para otras aplicaciones, este aprendizaje no sólo no tiene importancia, sino que es indeseable. [9] Otra de las razones por la cual no existe una definición única para el concepto de agente es debido a que los autores normalmente relacionan la definición al dominio de la aplicación, a las formas de cooperación y a los niveles de autonomía. [4]

Un Agente Inteligente es, desde un punto de vista ideal, aquel que responde al ambiente con la mejor decisión posible en una situación dada. Según Russell \& Norvig, un agente es un sistema capaz de percibir a través de sensores las informaciones del ambiente donde está insertado y reaccionar a través de efectores. Un agente se puede definir como una entidad de software que exhibe un comportamiento autónomo, que está situado en algún ambiente sobre el cual es capaz de realizar acciones para alcanzar sus propios objetivos de proyecto y a partir del cual percibe alteraciones. Posee un conjunto de propiedades específicas asociadas a su papel en la sociedad multiagente donde está insertado. Deben ser racionales, de tal forma que tomen la mejor decisión posible en un momento dado.

Ya existe consenso en que un agente debe tener como mínimo: autonomía (su comportamiento debe estar definido por su propia experiencia además de sus percepciones, sin limitarse a ninguno de los dos), reactividad y habilidad social (comunicación con otros agentes del ambiente). En un abordaje clásico para el área de agentes, la definición de Wooldridge, visualiza un agente como una entidad con capacidad de resolución de problemas encapsulada. En este contexto, un agente tiene las siguientes propiedades [6]:

- Autonomía: ejecuta la mayor parte de sus acciones sin interferencia directa de agentes humanos o de otros agentes computacionales, teniendo control total sobre sus acciones y estado interno;

- Habilidad social: interactúa con otros agentes (humanos o computacionales) para completar la resolución de sus problemas o para auxiliar a otros agentes;

- Capacidad de reacción: percibe y reacciona a las alteraciones del ambiente en que está insertado;

- Capacidad pro-activa: agentes, de tipo deliberativo, además de actuar en respuesta a las alteraciones ocurridas en su ambiente, presentan un comportamiento orientado a objetivos, tomando iniciativas cuando lo consideran apropiado.

Otras capacidades tomadas de la inteligencia humana que también pueden mostrar los agentes son [1]:

- Creencia, deseos, intenciones, obligaciones

- Veracidad (no engañar)

- Benevolencia (no entrar en conflicto y hacer lo que se le dice)

- $\quad$ Racionalidad (actuar según sus metas) 
- $\quad$ Movilidad (poder desplazarse físicamente)

Siendo un agente una entidad que encapsula conocimiento sobre algún dominio, nada más natural que agrupar agentes que poseen parte del conocimiento involucrado en la estrategia de resolución de un problema y que, a partir de eso, interactúen con el objetivo de complementar sus habilidades. Así, de la misma forma que en el mundo real existen empresas con empleados poseedores de diferentes habilidades y que, utilizando esas habilidades, desarrollan parte de las actividades necesarias al proceso productivo, se puede componer una sociedad de agentes donde para cada agente se proporcione un subconjunto de las habilidades requeridas por la estrategia de solución, donde a cada uno se le asigne parte de las tareas a ser cumplidas, de acuerdo con su disponibilidad de recursos. A este agrupamiento se le denomina SMA. [7] En el paradigma de los SMA, se aplican conceptos como la cooperación, negociación y especialización a las interacciones entre los agentes, para conseguir una meta común. Si puede compararse un agente con un ser humano, en el manejo de una función, de la misma manera puede compararse un sistema multiagente con un grupo de trabajo humano. [6]

\section{Consideraciones del Proyecto de un SMA}

Las características del abordaje multiagente impone necesidades que deben ser viabilizadas para que el sistema pueda ser considerado eficaz. En un SMA, se debe considerar criterios que viabilicen y garanticen la coherencia de las acciones de los agentes para alcanzar de manera efectiva los objetivos del SMA. Moulin y Chaib-Draa proponen un marco de trabajo que ofrece una estructura de análisis y clasificación de la mayor parte de las actividades de investigación en SMA, del cual se pueden citar dos perspectivas [7]:

- Perspectiva del Agente, enfoca elementos que caracterizan el agente involucrado en un SMA. Son ellos: categorías de agente, estructura y mantenimiento del conocimiento, habilidades de razonamiento, habilidades de adaptación y aprendizaje, y arquitecturas de agente.

- Perspectiva de Grupo, reúne aspectos de grupo, tales como: organización, coordinación, cooperación, negociación, comportamiento coherente, planeamiento, comunicación e interacción.

Dentro de la perspectiva de grupo, se puede definir tres grandes grupos de aspectos a ser considerados en el projecto de un SMA [7]:

- Aspectos Fundamentales, definen las características que deben ser viabilizadas para garantizar la compatibilidad entre las acciones de los agentes que constituyen el SMA.

- Aspectos Arquitecturales, definen las características que deben ser suministradas por la arquitectura a ser adoptada para la viabilización de los aspectos fundamentales dentro del SMA.
- Aspectos Ambientales, definen las características del ambiente en el cual se insertarán los agentes del SMA para que se pueda determinar los tipos de técnicas de percepción que deban ser utilizadas por estos agentes.

Aspectos Fundamentales

Los aspectos fundamentales a ser considerados en el desarrollo de un SMA para garantizar la compatibilidad de las acciones de los agentes son estructura, organización y coordinación. [7]

Actualmente, adoptar la idea de organización y de cambios organizacionales es importante para crear SMA más adaptables. No obstante, es necesario distinguir entre los términos estructura y organización. Por estructura se entiende el patrón de relaciones de información y control entre agentes, así como la distribución de las habilidades entre ellos. Así, la estructura ofrece una visualización de cómo el grupo resuelve los problemas y el papel que cada agente desempeña dentro de esta estructura, o sea, define los papeles y los relacionamientos para atender a las siguientes condiciones:

- Cobertura: cualquier habilidad necesaria para la resolución del problema debe estar insertada en el rol de habilidades de al menos un agente.

- Conectividad: los agentes deben interactuar de manera de permitir que sus habilidades sean integradas y desempeñadas en el sentido de contribuir a una solución global.

- Potencialidad: cobertura y conectividad deben ser alcanzables dentro de limitaciones computacionales y de comunicación, así como las especificaciones de confiabilidad del grupo.

De esta manera, cada agente desempeña uno o más papeles específicos en el sistema. Se define como papel aquello que se espera que el agente haga dentro de la organización, o sea, un conjunto de responsabilidades bien definidas dentro del contexto global del sistema que el agente puede cumplir con un cierto grado de autonomía.

El concepto de organización se refiere al conjunto de compromisos globales, creencias mutuas, e intenciones comunes a los agentes cuando actúan juntos para alcanzar un determinado objetivo. Estos elementos definen un conjunto de directrices a seguir por cada uno de los agentes del SMA, describiendo una política de interacción entre los agentes (p.ej.: el agente acredita que el agente tiene autoridad para controlar determinado recurso). Estas directrices pueden evitar la ocurrencia de situaciones caóticas en el SMA, creando patrones de comportamiento que eviten que el SMA se torne una "tierra sin ley", facilitando y también viabilizando la realización de los objetivos globales de la sociedad.

En la visión de Bond y Gasser, un sistema multiagente presupone coordinación entre un conjunto existente de agentes autónomos e inteligentes. Fundamentalmente, está involucrada la búsqueda de una funcionalidad en este sistema que permita que los agentes puedan coordinar sus conocimientos, objetivos, habilidades y planes individuales de una forma conjunta, en favor de la ejecución de una acción o de la resolución de algún problema. "Coordinación es el 
proceso por el cual un agente razona sobre sus acciones locales y las acciones (anticipadas) de otros agentes con el objetivo de garantizar que la comunidad funcione de manera coherente".

La definición de estrategias que concilien los intereses individuales de cada agente para que las actividades relacionadas se desarrollen de modo coordinado es uno de los aspectos fundamentales a ser considerados en el proyecto de SMA. La necesidad de coordinación entre múltiples agentes surge del hecho de que:

- existen dependencias entre las acciones de los agentes, o sea, la acción de un agente puede ser prerrequisito de la acción de otro agente; y

- ningún individuo tiene competencia, recursos o información suficientes para resolver un problema completo de forma independiente; donde

- debe ser garantizado el respeto a las restricciones globales a la solución del problema; y

- deben ser viabilizados procedimientos que garanticen la armonía durante la ejecución de una tarea de forma conjunta por más de un agente.

La coordinación es un factor vital para el funcionamiento de un SMA, pues sin coordinación los beneficios obtenidos de la resolución distribuida de problemas desaparecen y la comunidad puede degenerar en una caótica colección de individuos que actúan de forma inconexa en relación al sistema como un todo. Para eso, basta que un único agente tenga una visión parcial o imprecisa del sistema y que sus acciones puedan interferir en las acciones de otros agentes en lugar de soportarlas.

Durfee y otros identifican tres factores básicos que deben estar presentes para una coordinación con éxito:

- existencia de una estructura que permita a los agentes interactuar de forma predictiva;

- flexibilidades en las interacciones de tal forma que los agentes puedan operar en ambientes dinámicos y actuar satisfactoriamente con una visión parcial e imprecisa de la sociedad;

- los agentes deben poseer conocimiento y capacidad de razonamiento suficientes para explorar esta estructura y flexibilidad.

La manera más fácil de garantizar un comportamiento coherente dentro de un SMA es implantar un agente que tenga una perspectiva más amplia del sistema, actuando como un coordinador que, reuniendo informaciones sobre toda la sociedad, seria responsable por crear planes y atribuir tareas a los miembros de esta sociedad. No obstante, éste no es un abordaje práctico en sistemas reales por ser muy difícil crear un agente que se mantenga informado sobre todas las intenciones y creencias de todos los agentes del grupo. Además, un coordinador centralizado se convertiría en un cuello de botella de comunicación, que degradaría la performance del sistema. También se debe considerar el hecho de que, en este abordaje, una falla del agente coordinador comprometería el funcionamiento de todo el sistema, a pesar de que, en este caso, podrían ser adoptados mecanismos de tolerancia a las fallas donde otro agente podría asumir el papel de coordinador. A partir de eso, una problemática presentada para el proyecto de SMA pasa a ser el mantenimiento de la coherencia global sin un control global explícito. Según Oliveira, "los agentes deben razonar respecto a las acciones, pero también sobre el proceso de coordinación en si". Muchas investigaciones tienen su foco en el desarrollo de comunidades en las cuales el control y los datos son distribuidos. Una desventaja proveniente de la distribución del control y de los datos es la dificultad de tener conocimiento sobre el estado global del sistema, que está disperso a través de la comunidad, puesto que cada individuo posee una visión parcial e imprecisa de esta perspectiva.

Los principales requisitos para la coordinación son:

- comunicación entre los agentes;

- reconocimiento de las interacciones potenciales entre planes; $y$

- negociación entre los agentes.

Aspectos Arquitecturales

Se puede utilizar una taxonomía para clasificar las posibles formas de disponer los relacionamientos existentes entre los agentes [7]:

- Jerárquica: dispone los agentes en una estructura jerárquica en la cual la comunicación ocurre de forma jerárquica. En estos casos, cada agente puede comunicarse solamente con los agentes supervisados por él o por su propio supervisor. Este tipo de estructura: (i) dispensa mecanismos para localización de agentes y (ii) reduce significativamente la cantidad de comunicación en el sistema, pero (iii) presenta una estructura que no permite que los agentes se organicen dinámicamente para atender mejor las necesidades de una tarea específica (iv) donde los niveles más bajos dependen de los niveles más altos, y los niveles más altos pueden tener control parcial o total en relación a los niveles más bajos.

- Nivelada: implica que cada agente puede contactar directamente con cualquiera de los otros agentes. De esta forma, pueden crear estructuras de manera dinámica para realizar una tarea específica.

- Agentes Compuestos por Agentes: presupone la existencia de algunos agentes que son componentes de otros agentes.

- Sistemas Multiagentes Compuestos por Sistemas Multiagentes: considera un SMA como un agrupamiento de varios módulos que, a su vez, son vistos aisladamente como SMA.

Aspectos Ambientales

Según la definición de Wooldridge, un agente está situado en algún ambiente. D'Amico y otros entienden que el ambiente puede ser visto como todo lo que rodea a un agente. Así, el ambiente constituye el contexto donde ocurren todas las interacciones entre los agentes. A través del ambiente ocurre la dispersión del control, de los datos y del conocimiento por la comunidad de agentes. "Una organización no garantiza su supervivencia a partir de su capacidad de aprendizaje y de ajuste a las características y cambios en el mundo". Por tanto, es imprescindible que los agentes de una organización posean conocimiento sobre las alteraciones ocurridas en el ambiente, pues la ausencia 
de esta capacidad inviabiliza la adaptación a la nueva realidad.

No obstante, un factor importante a considerar en el proyecto de un SMA es el tipo de ambiente en el cual sus agentes estarán situados, que determina cual es el tipo de representación del ambiente sobre el cual cada uno de los agentes deberá trabajar, así como la manera de actuar y de percibir las alteraciones en el ambiente. Las principales clasificaciones a ser hechas en un ambiente son [7]:

- Accesible x Inaccesible: esa característica determina si el aparato sensorial del agente le ofrece un estado completo del ambiente. Si esto ocurre, el ambiente es considerado accesible; caso contrario, es considerado inaccesible. Un ambiente es considerado efectivamente accesible si los sensores detectan todos los aspectos relevantes para la elección de la acción. En el caso de que un ambiente sea accesible, no es necesario que el agente mantenga cualquier representación interna del mundo.

- Determinístico x No-Determinístico: si el estado del ambiente es determinado solamente por su estado actual y las actuaciones de los agentes, se puede decir que este ambiente es determinístico.

- Episódico x No-Episódico: en un ambiente episódico la experiencia del agente está dividida en episodios. Cada uno de ellos consiste en percepciones y acciones de los agentes, y la calidad de cada acción depende solamente del episodio en si.

- Estático x Dinámico: un ambiente es dinámico si puede cambiar cuando el agente está deliberando; en caso contrario es estático. Un ambiente se denomina semi-estático cuando no cambia con el transcurso del tiempo, sólo con las acciones desempeñadas por los agentes.

- Discreto x Continuo: se llama discreto al ambiente que tiene un número limitado de percepciones y acciones distintas y claramente definidas.

\section{Modelado de STI con SMA}

Los Sistemas Tutores Inteligentes, como sistemas educacionales proyectados para ofrecer algún tipo de auxilio personalizado al alumno, considerando el proceso de aprendizaje de un determinado contenido, involucran aspectos de Ingeniería del Software y no aspectos meramente pedagógicos en su modelado e implementación. Los avances más recientes en el campo de los ambientes de aprendizaje inteligentes, han propuesto el uso de arquitecturas basadas en sociedades de agentes para ampliar las posibilidades de realizar esa asistencia personalizada al alumno. Los principios de los sistemas multiagentes han mostrado un potencial bastante adecuado al desarrollo de sistemas de enseñanza, debido a que por su naturaleza, el problema de la enseñanza y del aprendizaje puede ser resuelto más fácilmente de forma cooperativa. [6]

La característica esencial del abordaje SMA es la filosofía de resolución distribuida de problemas, en la cual se adopta una estrategia de dividir para conquistar.
La resolución cooperativa distribuida de problemas consiste en que un problema se divide en subproblemas y cada uno se soluciona separadamente por medio de un agente, cada uno de ellos comunicándose o cooperando entre si cuando fuera necesario, con la idea básica de que la suma de los resultados locales corresponderá a la solución del problema general. [4]

El uso de agentes en la concepción de sistemas educacionales puede aportar algunas ventajas como: reacción a las acciones del usuario, credibilidad, modelado de sistemas cooperativos multiusuarios y modularidad, por el hecho de que cada agente es un módulo único e independiente del otro, siendo más fácil adicionar otros agentes a estos sistemas. Además, ambientes de enseñanza basados en arquitecturas multiagentes posibilitan el soporte del desarrollo de sistemas de forma más robusta, más rápida y con menos costos.

\section{Justificación}

La aplicación de la tecnología de agentes en la concepción de Sistemas de Información está justificada cuando el problema posee las siguientes características:

- El dominio involucra distribución intrínseca de los datos, capacidad de resolución de problemas y responsabilidades.

- Necesidad de mantener la autonomía de las subpartes, sin una pérdida de la estructura organizacional.

- Complejidad en las interacciones, incluyendo negociación, información compartida y coordinación.

- Imposibilidad de descripción de la solución del problema a priori, debido a la posibilidad de perturbaciones en tiempo real en el ambiente y procesos de negocio de naturaleza dinámica.

Por tanto, los softwares educacionales pueden utilizar la tecnología de agentes porque poseen todas esas características.

\section{Ventajas}

Las propuestas de utilización de arquitecturas SMA en STI aportan una gran ventaja en relación a las arquitecturas tradicionales de STI por presentar flexibilidad en el tratamiento de los elementos que componen el sistema. Además, el hecho de usar agentes para modelar sus componentes, posibilita el agrupamiento de la arquitectura tradicional (un módulo $=$ un agente) o la explosión de cada módulo en varios agentes.

Se puede considerar que las ventajas son:

- El conocimiento puede distribuirse entre varios "tutores", cada uno con sus creencias, deseos, objetivos, emociones y planes de acción. Esta distribución crea mayores oportunidades de variar técnicas pedagógicas.

- El aprendiz interactúa con un tutor de forma más flexible. 
- El aprendiz puede comunicar conocimientos al tutor que serán comunicados a otros aprendices.

Las metodologías multiagentes han surgido como una alternativa para concebir aplicaciones de aprendizaje distribuido, debido al conjunto de características inherentes al concepto de SMA y a las peculiaridades de una sociedad de agentes. La razón principal es el hecho de que esta tecnología lidia muy bien con aplicaciones críticas, tales como: distancia, cooperación entre diferentes entidades e integración de diferentes componentes de software. [6]

\section{Propuesta de Diseño}

El diagrama de clases entidad de la Figura 1 modela la información maestra del sistema, relativa al dominio de conocimiento o situación del sistema tutorial.

Para cada alumno registrado en el sistema los agentes del módulo de interacción registrarán referencias a estos datos como información transaccional, es decir, para reflejar las veces que el alumno accedió a cada material (agente monitor de navegación) y características de su interacción con el mismo (agente monitor de concentración), así como la calificación de las evaluaciones que se le hayan efectuado (agente de registo de evaluaciones).

Cabe destacar que este modelo permite evaluar tanto conocimientos como habilidades, y tanto en función de la organización temática del domino como en función de cuánto ha aprehendido el alumno los principios fundamentales del campo disciplinar, que cruzan horizontalmente los temas.

El módulo tutor o de ofrecimientos se organiza con un conjunto de agentes representantes de distintas estrategias de enseñanza, compuestos a su vez por un agente del saber y otro del saber hacer, que negociarán sus interacciones en función de la evolución de cada alumno.

Cada agente tutor analizará la información registrada para un alumno cuando éste se conecte, y en función de su interacción con el sistema en la sesión actual y del historial de interacciones negociará con el resto de los agentes tutores su participación para presentarle material o evaluaciones.

La clase asociación Inherencia se caracteriza por un escalar entre 0 y 1 que represente el grado de compromiso de un material con el principio asociado.

La clase asociación Importancia se caracteriza por un valor escalar entre 0 y 1 que represente el grado de importancia o de contribución de un material con el tema asociado.

Preguntas y Problemas se clasifican como material, ya que pueden ser presentadas al alumno junto con las respuestas y soluciones acertadas o alternativas como una estrategia de enseñanza por casos por parte de un agente tutor. Pero también un agente tutor puede presentar una pregunta o problema junto con respuestas o soluciones alternativas para evaluar cuánto sabe o cuánto sabe hacer el alumno sobre un tema.

La clase asociación Evaluación se caracteriza por un valor escalar que represente la corrección $u$ optimalidad de una respuesta o solución con una pregunta o problema, y la asociación Violación con Principio indica si una respuesta viola a un principio.

\section{Arquitectura}

El módulo de dominio o situación se constituye por las clases entidad y por clases de control para orientar la carga de material didáctico que permitan evaluar la consistencia y completitud de los datos.

El módulo tutor o de ofrecimientos se constituye por clases de control que implementan agentes, a razón de un agente por modelo de aprendizaje que se desee considerar.

El módulo de interacción se constituye por clases entidad que agrupan historiales de acceso y de evaluación, y clases de control para implementar los agentes monitores de navegación y concentración, de registro de evaluaciones y perfilador de estudiantes; este último evalúa los registros sobre cada alumno para determinar su perfil de aprendizaje y, en cada sesión de un alumno darle intervención a los agentes tutores más adecuados.

\section{Conclusiones y Trabajos Futuros}

Se ha diseñado un sistema según el estado actual de las tecnologías involucradas donde la innovación radica en la organización o diseño del contenido y en la definición de los agentes. Se preve desarrollar el sistema en entorno web y utilizarlo como complemento de la enseñanza y monitoreo de alumnos para los cursos de la asignatura Computación donde cumplen funciones los autores. 


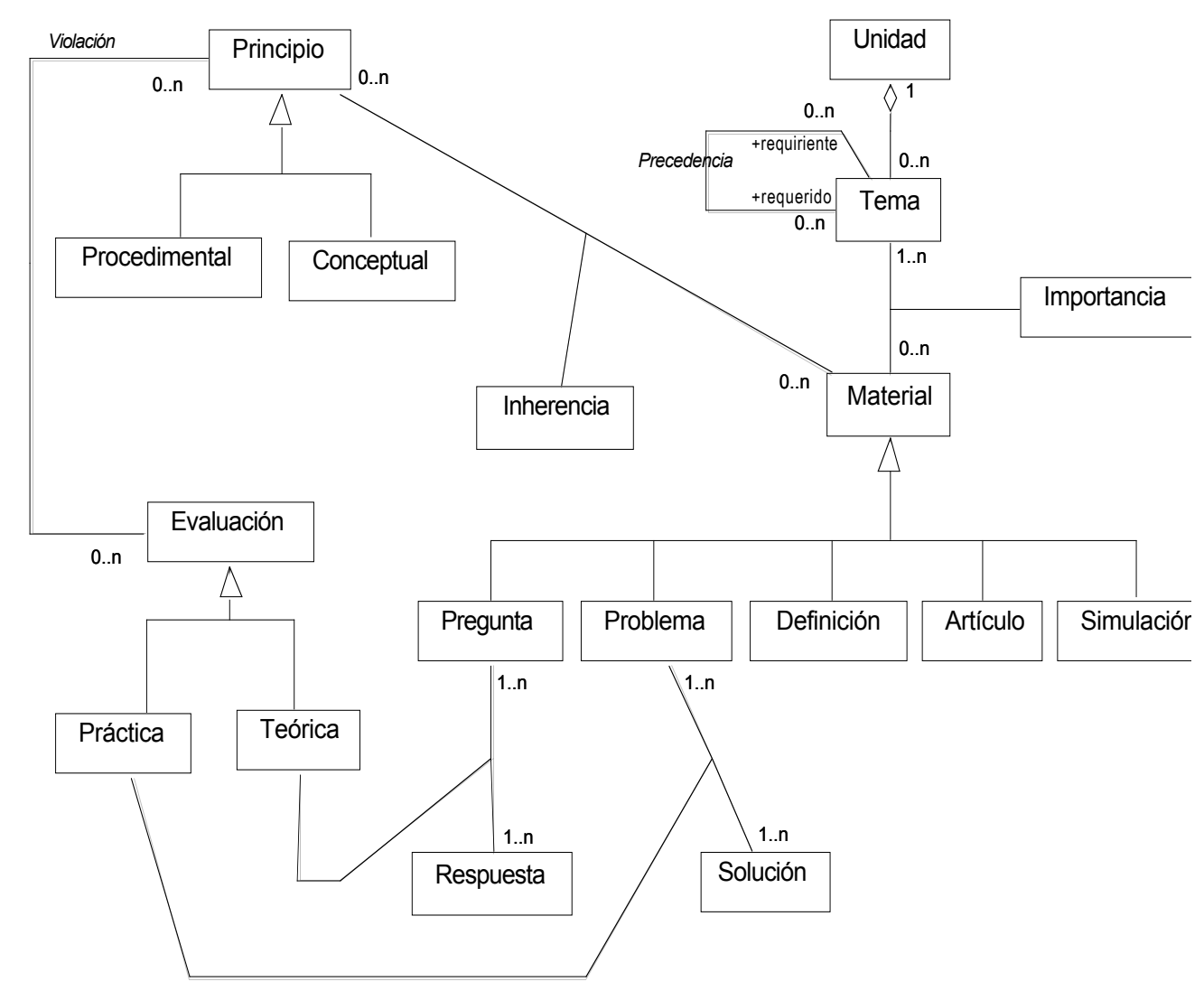

Figura 1

\section{Referencias}

[1]. BEJÁR, J. Introducción A los agentes y arquitecturas. Facultad d' Ínformàtica de Barcelona Universirat Politècnica de Catallunya. Aplicaciones de la IA - Curso 2004/2005 $1^{\circ}$ cuatrimestre.

[2]. BOLZAN, W., Martins Giraffa, L.M. 2002. Estudo Comparativo sobre Sistemas Tutores Inteligentes Multiagentes Web. Techical Report Series. Number 024. Faculdade de Informática. PUCRS. Brazil.

[3]. COSTA, M. Sistemas Tutores Inteligentes. www.nce.ufrj.br/ginape/publicacoes/trabalhos/Mac arioMaterial/Sti.htm

[4]. FRIGO, L. B, POZZEBON, E., BITTENCOURT, G. 2004. O Papel dos Agentes Inteligentes nos Sistemas Tutores Inteligentes. World Congress on Engineering and Technology Education. March 14-17. São Paulo. Brazil.

[5]. HOGG, Tad, HUBERMAN, Bernardo A.

Controlling Chaos in Distributed System. IEEE Trans. on Systems, Man and Cybernetics 21, 13251332 (1991)
[6]. JIMÉNEZ REY, E., GROSSI, M. D., PERICHINSKY, G. 2005. Una Aplicación de la Tecnología de MultiAgentes a los Sistemas Tutores Inteligentes: Enseñanza de Computación en Carreras de Ingeniería. VII Workshop de Investigadores en Ciencias de la Computación WICC 2005. UNRC-REDUNCI. Mayo 13-14. Río Cuarto.

[7]. JUCHEM, M., Melo Bastos, R. 2001. Engenharia de Sistemas Multiagentes: Uma Investigacão sobre o Estado da Arte. Technical Report Series. Number 014. Faculdade de Informática. PUCRS-Brazil. 2001.

[8]. URRETAVIZCAYA LOINAZ, M. 2001. Sistemas Inteligentes en el ámbito de la Educación. Inteligencia Artificial, Revista Iberoamericana de Inteligencia Artificial. No.12 pp. 5-12. ISSN: 1137-3601.

[9]. WOOLDRIDGE, M. J. 2000. Multiagent Systems. En "Inteligents Agents" (Cap. 1, pp 2-3). G. Weiss Ed. 